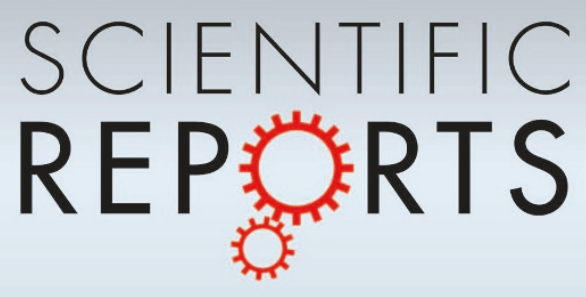

\section{OPEN}

SUBJECT AREAS:

SECONDARY

METABOLISM

GENETIC ENGINEERING

METABOLIC ENGINEERING

MOLECULAR ENGINEERING IN

PLANTS

Received

16 November 2012

Accepted

3 April 2013

Published

12 June 2013

Correspondence and requests for materials should be addressed to M.N. (mnishiha@ibrc. or.jp)

* Current address:

Department of

Biological and

Environmental

Science, Graduate

School of Agriculture,

Shizuoka University,

836 Ohya, Suruga-ku,

Shizuoka 422-8529,

Japan.

\title{
Genetic engineering of yellow betalain pigments beyond the species barrier
}

\author{
Takashi Nakatsuka'*, Eri Yamada' ', Hideyuki Takahashi' ${ }^{1}$, Tomohiro Imamura' ', Mariko Suzuki' ${ }^{2}$ \\ Yoshihiro Ozeki², Ikuko Tsujimura', Misa Saito', Yuichi Sakamoto', Nobuhiro Sasaki² \\ \& Masahiro Nishihara'
}

\begin{abstract}
'Iwate Biotechnology Research Center, 22-174-4 Narita, Kitakami, Iwate 024-0003, Japan, ${ }^{2}$ Department of Biotechnology and Life Science, Faculty of Engineering, Tokyo University of Agriculture and Technology, 2-24-16 Naka-cho, Koganei, Tokyo 184-8588, Japan.
\end{abstract}

Betalains are one of the major plant pigment groups found in some higher plants and higher fungi. They are not produced naturally in any plant species outside of the order Caryophyllales, nor are they produced by anthocyanin-accumulating Caryophyllales. Here, we attempted to reconstruct the betalain biosynthetic pathway as a self-contained system in an anthocyanin-producing plant species. The combined expressions of a tyrosinase gene from shiitake mushroom and a DOPA 4,5-dioxygenase gene from the four-o'clock plant resulted in successful betalain production in cultured cells of tobacco BY2 and Arabidopsis T87. Transgenic tobacco BY2 cells were bright yellow because of the accumulation of betaxanthins. LC-TOF-MS analyses showed that proline-betaxanthin (Pro-Bx) accumulated as the major betaxanthin in these transgenic BY2 cells. Transgenic Arabidopsis T87 cells also produced betaxanthins, but produced lower levels than transgenic BY2 cells. These results illustrate the success of a novel genetic engineering strategy for betalain biosynthesis.

r he coloration of flowers and fruits is due to the accumulation of pigments such as flavonoids (including anthocyanins), carotenoids, and betalains ${ }^{1,2}$. Anthocyanins and carotenoids are widely distributed in angiosperms, whereas betalains are water-soluble nitrogenous pigments found only in some plants in the order Caryophyllales (but not in the families Caryophyllaceae and Molluginaceae) ${ }^{3}$ and in some higher fungi, such as fly agaric (Amanita muscaria $)^{4}$. Although betalains have functions analogous to those of anthocyanins as pigments, anthocyanins and betalains are mutually exclusive pigments in plants ${ }^{3}$. This raises the taxonomic mystery of why betalains cannot be produced in any plants outside of the order Caryophyllales ${ }^{5,6}$.

Betalains, which include the red-violet betacyanins and the yellow betaxanthins, are biosynthesized from the amino acid tyrosine by several enzymatic and spontaneous chemical steps (Fig. 1) ${ }^{7}$. The first step is the conversion of tyrosine to dihydroxyphenylalanine (L-DOPA) by uncharacterized enzyme(s) with tyrosine-hydroxylating activity. L-DOPA is converted to betalamic acid (BA), which is an important precursor of betalain biosynthesis, by DOPA 4,5-dioxygenase (DOD) ${ }^{8}$. In addition, L-DOPA is also converted to cyclo-DOPA by a cytochrome P450 enzyme ${ }^{9}$. BA conjugates with an amino acid or amine to form yellow betaxanthins. This condensation of BA with amino acids is considered as a spontaneous non-enzymatic reaction ${ }^{10}$. There are two pathways postulated for betacyanin biosynthesis (Fig. 1). Namely, glycosylation steps occur at the precursor molecule, cyclo-DOPA, or after the condensation of cyclo-DOPA with BA to form betanidin. Both betanidin 5-O-glucosyltransferase (betanidin 5GT) and cyclo-DOPA 5-O-glucosyltransferase (cDOPA 5GT) have been identified ${ }^{11,12}$. Both betacyanins and betaxanthins accumulate in the vacuole, like anthocyanins ${ }^{2}$. As described above, the first enzymatic step in betalain biosynthesis is the formation of L-DOPA from tyrosine, which involves a hydroxylation step (Fig. 1). In plants, tyrosine-hydroxylating activity has mainly been attributed to tyrosinase, but has also been attributed to tyrosine hydroxylase ${ }^{13-16}$. However, the involvement of either enzyme has not been shown conclusively, nor has any gene encoding a product with this activity been yet identified in betalain-producing plants. In contrast, the tyrosinase involved in the formation of melanin, the brown pigment produced during development and post-harvest storage of mushrooms, has been well studied and characterized ${ }^{17}$.

Schliemann et al. ${ }^{18}$ proposed that the concerted action of two enzymes, DOD from A. muscaria and DOPA oxidase from Portulaca grandiflora, synthesized betanidin, one of the betacyanins, in vitro. Feeding of betalamic acid to broad bean (Vicia faba L.) seedlings, which do not synthesize betaxanthins, resulted in the formation of dopaxanthin ${ }^{10}$. More recently, Harris et al. ${ }^{19}$ reported that transgenic Arabidopsis expressing the DOD gene from 


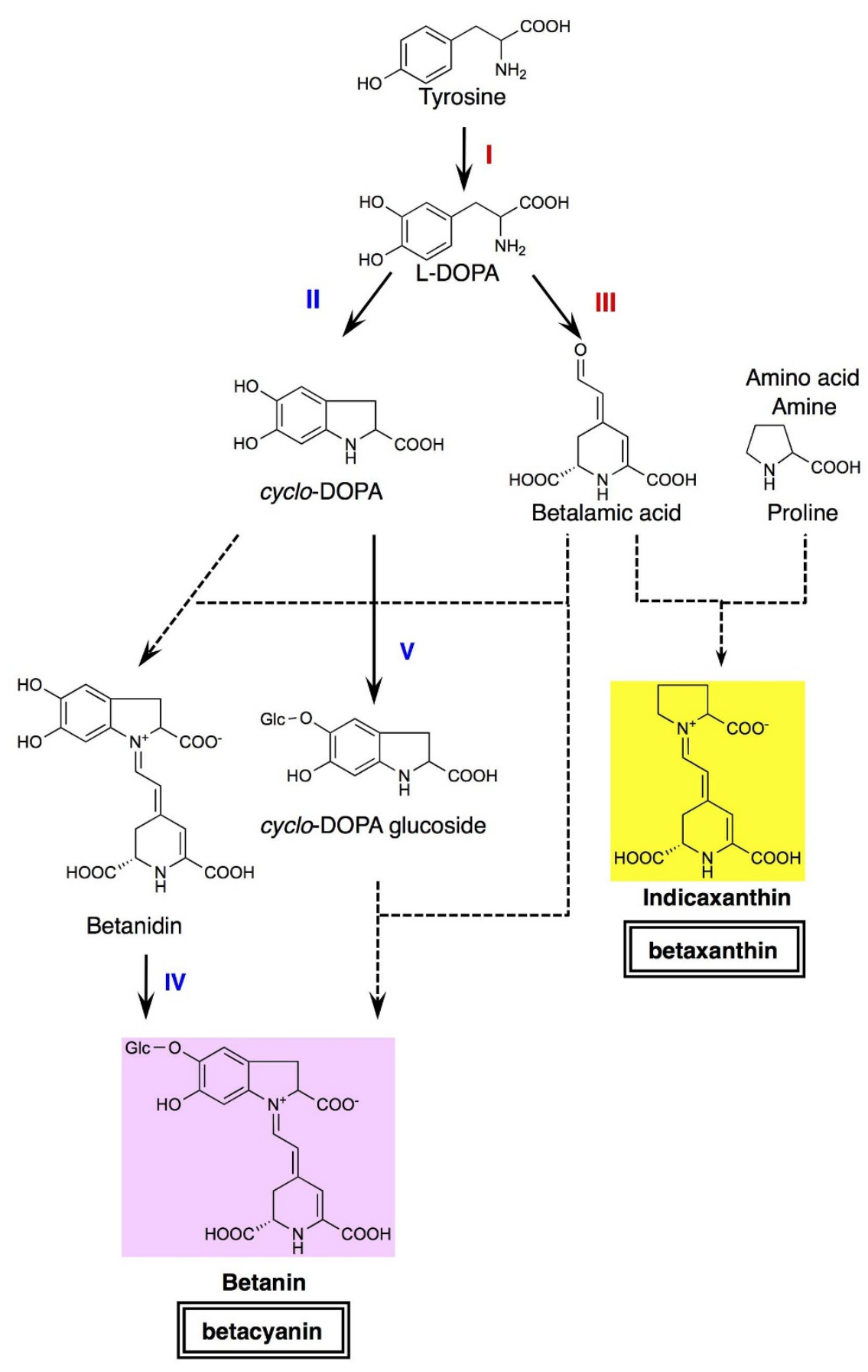

Figure 1 Betalain biosynthetic pathway. Thick arrows show enzymatic reactions, dotted arrows show spontaneous reactions. I, enzyme with tyrosinehydroxylating activity (LeTYR in this study); II, L-DOPA oxidase; III, DOPA 4,5-dioxygenase (MjDOD in this study); IV, betanidin 5-O-

glucosyltransferase; V, cyclo-DOPA 5-O-glucosyltransferase. Enzymes used for betaxanthin engineering in this study are shown in red font.

the fungus $A$. muscaria produced betaxanthin and betacyanin when supplied with the substrate L-DOPA. However, there are no reports on production of betalain pigments in non-betalain producing plants in vivo without feeding of the pathway intermediates.

In this study, we have shown that production of betaxanthin pigments without exogenous substrates is possible in suspensioncultured cells of both tobacco and Arabidopsis. We demonstrated that the combined expressions of a fungal tyrosinase gene and a plant $D O D$ gene made a successful heterologous expression system for production of betaxanthins in these cultured cells. This is the first report of a self-contained betalain biosynthetic system in plant species outside of the order Caryophyllales.

\section{Results}

Establishment of transgenic BY2 suspension cells for production of betaxanthins. To produce betalain pigments in anthocyaninproducing plant species outside of the order Caryophyllales, we first selected tobacco (Nicotiana tabacum), which belongs to the family Solanaceae, as the transformation host. The tobacco BY-2 cell line is the most popular suspension cell line in plant research and its rapid growth characteristics make it an ideal experimental material ${ }^{20}$. We produced transgenic tobacco BY2 cells expressing tyrosinase from shiitake mushroom (Lentinula edodes, LeTYR) ${ }^{21}$ and/or $M j D O D$ from the four-o'clock plant (Mirabilis jalapa) ${ }^{22}$ under the control of the cauliflower mosaic virus $35 \mathrm{~S}$ promoter 
(35Sp). Calli expressing both 35Sp-LeTYR and 35Sp-MjDOD showed bright yellow coloration (Fig. 2a). Transgenic calli expressing 35Sp-LeTYR or 35Sp-MjDOD showed no color change compared with those of the vector control and untransformed wild-type (Fig. 2a).

Expression and enzymatic activity analysis of transgenic tobacco BY2 cells. We analyzed transgene expressions in each of the three transgenic BY2 lines by northern blot analysis (Fig. 2b). The expressions of LeTYR were detected in both 35Sp-LeTYR and 35Sp-LeTYR/35Sp-MjDOD transgenic BY2 calli. However, the expression levels of LeTYR in 35Sp-LeTYR/35Sp-MjDOD lines were higher than those in 35Sp-LeTYR lines (Fig. 2b). In addition, we detected degraded and aberrant LeTYR transcripts in transgenic BY2 calli. These degraded and aberrant transcripts of LeTYR were also observed in the fruiting body of shiitake mushroom ${ }^{21}$. There were similar expression levels of $M j D O D$ in $35 \mathrm{Sp}-\mathrm{MjDOD}$ and 35Sp-LeTYR/35Sp-MjDOD lines, except for 35Sp-MjDOD line no. 1. Western blot analysis of the tyrosinase protein with an anti-LeTYR polyclonal antibody was performed for transgenic BY2 calli (Fig. 2c). In the crude protein extracts from shiitake mushroom, there were two bands corresponding to the premature $68-\mathrm{kDa}$ and mature 45 $\mathrm{kDa}$ proteins, as reported by Sato et al. ${ }^{21}$. In transgenic BY2 calli with $35 \mathrm{Sp}-\mathrm{LeTYR}$ or $35 \mathrm{Sp}-\mathrm{LeTYR} / 35 \mathrm{Sp}-\mathrm{MjDOD}$, premature $68-\mathrm{kDa}$ signals were detected, but mature $45-\mathrm{kDa}$ signals were scarcely detected. The $68-\mathrm{kDa}$ signals in $35 \mathrm{Sp}$-LeTYR lines were much weaker than those in 35Sp-LeTYR/35Sp-MjDOD co-expressing lines (Fig. 2c). The results of western blot analysis were largely consistent with the expression levels determined by northern blot analysis (Figs. 2b, c).

Next, we investigated tyrosine-hydroxylating activities in $35 \mathrm{Sp}$ LeTYR and 35Sp-LeTYR/35Sp-MjDOD transgenic BY2 cell lines. The crude proteins extracted from transgenic BY2 lines showed no DOPA oxidase activity in the 3-methyl-2-benzothiazolinone hydrazone hydrochloride (MBTH) assay (data not shown). Thus, the tyrosine-hydroxylating activity, which synthesizes L-DOPA from tyrosine, was compared among the transgenic BY2 cell lines (Fig. 2c). No tyrosine-hydroxylating activity was detected in wildtype, the vector control, or 35Sp-MjDOD lines. Among the three
35Sp-LeTYR lines, line no. 3, which showed the strongest $68-\mathrm{kDa}$ signal in the western blot analysis, showed tyrosine-hydroxylating activity of $1.2 \mathrm{nmol} \mathrm{L-DOPA} \mathrm{h}^{-1} \mathrm{mg}_{\text {protein }}^{-1}$. The 35Sp-LeTYR/ 35Sp-MjDOD lines showed tyrosine-hydroxylating activities comparable to, or 3-fold higher than, that of 35Sp-LeTYR line no. 3 . These tyrosine-hydroxylating activities were largely consistent with the expression levels determined by northern blot and western blot analyses.

LC-TOF-MS analyses of betaxanthins in transgenic BY2 cells. Pigments accumulated in transgenic BY2 calli were investigated by LC-TOF-MS. We detected BA $(\mathrm{m} / \mathrm{z}=212.056, \lambda \max =425 \mathrm{~nm})$, the precursor of betaxanthin, in 35Sp-LeTYR/35Sp-MjDOD transgenic cell lines, but not in the vector control, 35Sp-LeTYR, or 35SpMjDOD lines. Therefore, the combined expression of LeTYR and $M j D O D$ was essential for accumulation of betalamic acid in tobacco BY2 cells.

35Sp-LeTYR/35Sp-MjDOD transgenic BY2 lines nos. 1, 2, and 3 accumulated $1.16 \pm 0.13,1.69 \pm 0.18$, and $1.67 \pm 0.05 \mu$ mole betaxanthins/g dry weight, respectively, as calculated from the molar extinction coefficient for betaxanthins. When 4-week-old calli were compared, transgenic line no. 3 showed the most stable proliferation and vivid yellow color among the three transgenic lines (Fig. 3a, Supplementary Fig. S1). The accumulation of betaxanthins started to increase at 4 weeks after subculture and reached the maximum at 8 weeks, thereafter decreasing rapidly (Fig. 3b). However, their calli turned brown after 5 weeks subculture, and became stunted (Fig. 3a, Supplementary Fig. S1). Therefore, 4-week-old calli of 35Sp-LeTYR/ 35Sp-MjDOD line no. 3 were used for further analyses.

HPLC analysis detected one major and some minor peaks at $470 \mathrm{~nm}$ (Fig. 3c). There was no clear peak in the vector control, 35Sp-LeTYR, or 35Sp-MjDOD transgenic BY2 calli. The major peak (retention time $41.3 \mathrm{~min}, \mathrm{~m} / \mathrm{z}=309.108, \lambda \max =480 \mathrm{~nm}$ ) was assigned to proline-Bx (Pro-Bx, indicaxanthin; Fig. 1) by comparison with synthesized betaxanthin standards. The $35 \mathrm{Sp}$-LeTYR/35SpMjDOD transgenic BY2 line no. 3 accumulated a maximum of $3.3 \mu$ moles Pro-Bx/g dry weight (Fig. $3 c$, peak 3), based on synthetic authentic Pro-Bx as the standard. The other minor peaks $1(23.8 \mathrm{~min}, \mathrm{~m} / \mathrm{z}=340.114, \lambda \max =470 \mathrm{~nm})$ and $2(27.4 \mathrm{~min}$, a

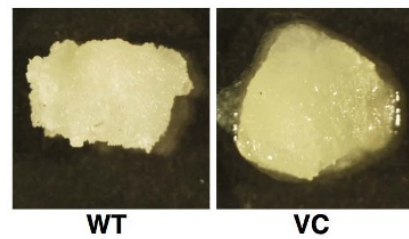

b

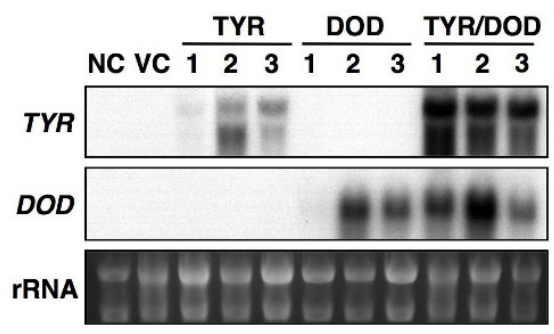

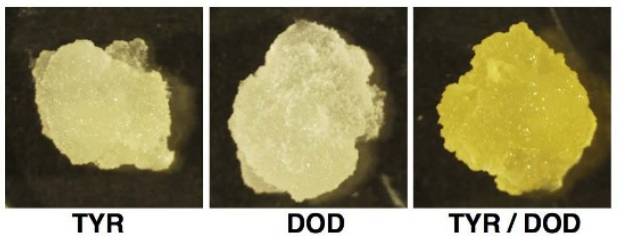

C

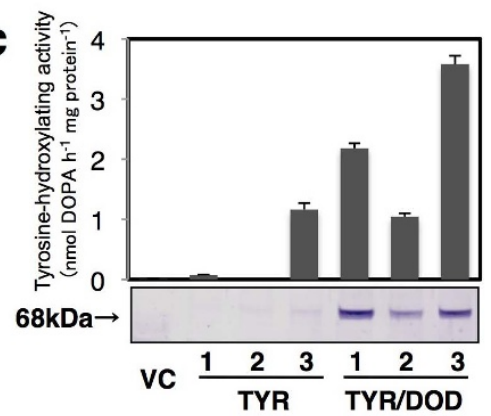

Figure $2 \mid$ Analyses of transgenic tobacco BY2 calli. (a) Images of typical transgenic BY2 calli. WT, wild-type; VC, vector control (pIG121-Hm); TYR, 35Sp-LeTYR line no. 3; DOD, 35Sp-MjDOD line no. 3; TYR/DOD, 35Sp-LeTYR/35Sp-MjDOD line no. 3. (b) Northern blot analysis. Total RNAs were isolated from wild-type (WT) and transgenic BY2 including vector control (VC), and three independent transgenic lines 35Sp-LeTYR (TYR), 35SpMjDOD (DOD), and 35Sp-LeTYR/35Sp-MjDOD (TYR/DOD). Total RNAs (5 $\mu \mathrm{g}$ ) were separated on an agarose gel and then transferred to a nylon membrane and hybridized with LeTYR or MjDOD probes. Ethidium bromide staining of rRNA bands shows quality and loading controls. (c) Tyrosinehydroxylating activity and accumulation of LeTYR proteins in transgenic BY2 calli. The activity was determined by CE-MS analysis as described in Materials and methods. Western blot analysis was performed using anti-LeTYR antibody ${ }^{21}$. 


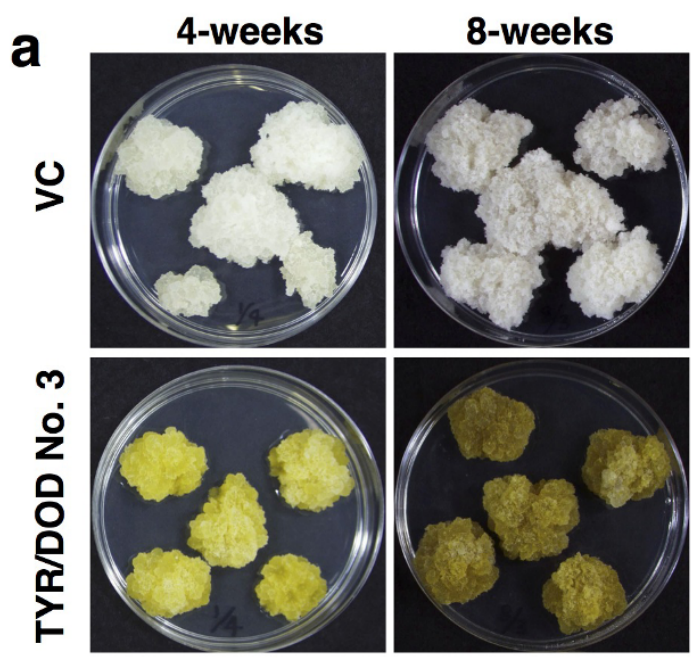

b

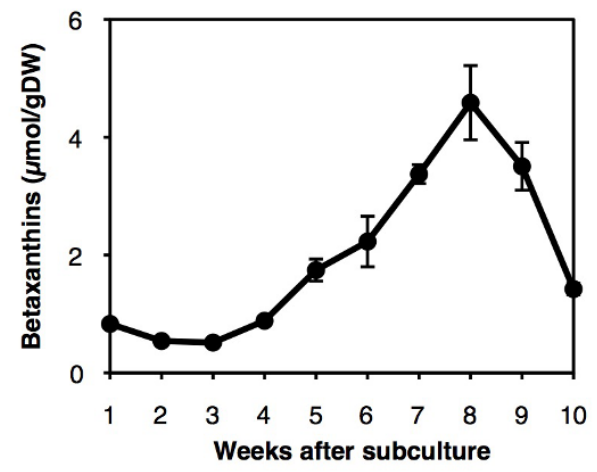

C

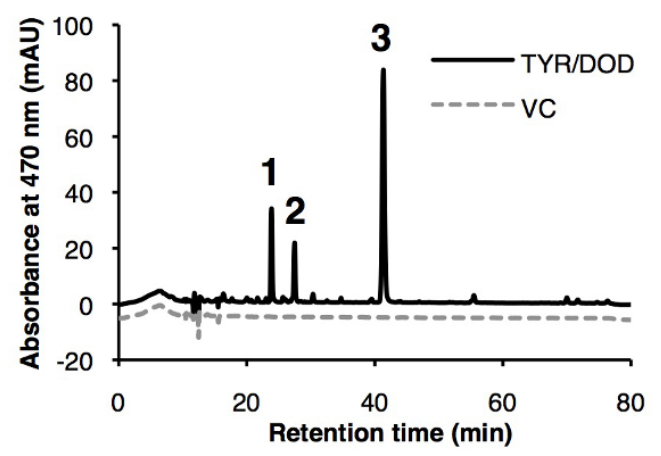

Figure 3 Betaxanthin accumulation in 35Sp-LeTYR/35Sp-MjDOD transgenic BY2 cells. (a) Typical phenotypes of vector control (VC) and 35Sp-LeTYR/35Sp-MjDOD line no. 3 (TYR/DOD no. 3) calli after 4and 8-weeks subculture. (b) Change in betaxanthin concentration over time in transgenic calli line no. 3 after subculture. Betaxanthins were extracted in $80 \%(\mathrm{v} / \mathrm{v})$ methanol containing $0.04 \%(\mathrm{v} / \mathrm{v}) \mathrm{HCl}$, and concentration was calculated from absorbance at $480 \mathrm{~nm}$. (c) Identification of betaxanthins by LC-TOF-MS in 35Sp-LeTYR/35SpMjDOD transgenic BY2 cells. Chromatography profiles of extracts from vector control (dashed line) and 35Sp-LeTYR/35Sp-MjDOD line no.3 (thick line). Peak 1, retention time $23.8 \mathrm{~min}, \mathrm{~m} / \mathrm{z}=340.114$, $\lambda \max =470 \mathrm{~nm}$, Glutamine-Bx (Vulgaxanthin I); Peak 2, $27.4 \mathrm{~min}$, $\mathrm{m} / \mathrm{z}=313.104, \lambda \max =470 \mathrm{~nm}$, Threonine-Bx; Peak 3, $41.3 \mathrm{~min}$, $\mathrm{m} / \mathrm{z}=309.1084, \lambda \max =480 \mathrm{~nm}$, Proline-Bx (Indicaxanthin).

$\mathrm{m} / \mathrm{z}=313.104, \lambda \max =470 \mathrm{~nm}$ ) were assigned to glutamine- $\mathrm{Bx}$ (Gln-Bx, vulgaxanthin I) and threonine-Bx (Thr-Bx), respectively (Fig. 3c). Accumulation of betaxanthins was also detected in other 35Sp-LeTYR/35Sp-MjDOD transgenic BY2 lines.
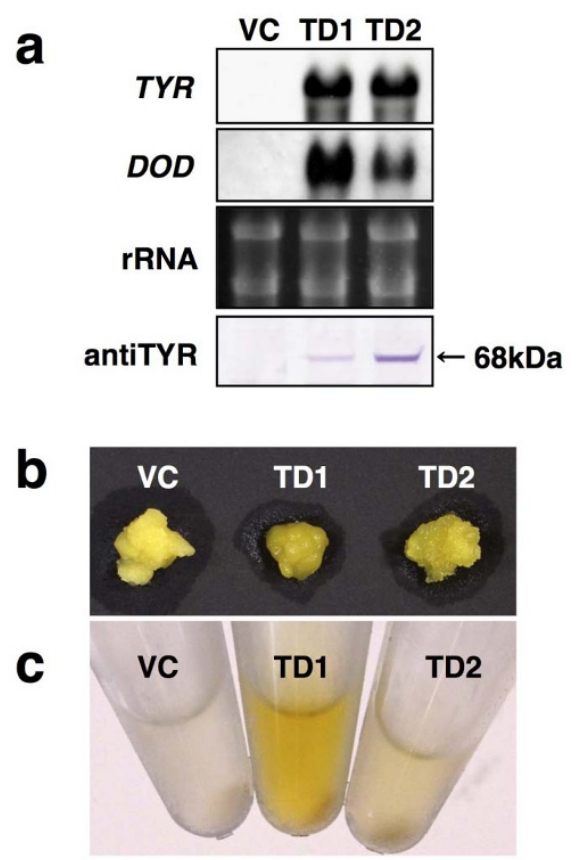

Figure 4 | Analysis of 35Sp-LeTYR/35Sp-MjDOD transgenic Arabidopsis T87 cells. (a) Analyses of $L e T Y R$ and MjDOD transcripts and LeTYR protein accumulation. VC, vector control pIG121Hm; TD1 and TD2, independent 35Sp-LeTYR/35Sp-MjDOD transgenic T87 cell lines. (b) Images of typical transgenic T87 calli. (c) Aqueous extracts from transgenic T87 calli.

Production of betaxanthins in Arabidopsis T87 cells. In addition to tobacco BY2 cells, we produced 35Sp-LeTYR/35Sp-MjDOD transgenic calli of Arabidopsis T87 cells. We obtained 40 independent transgenic calli with $35 \mathrm{SpLeTYR} / 35 \mathrm{SpMjDOD}$, and selected two transgenic calli lines that showed high expression levels of both LeTYR and MjDOD genes in a northern blot analysis (Fig. 4a). Western blot analysis detected $68-\mathrm{kDa}$ signals of the LeTYR protein in both transgenic lines, like in transgenic BY2 calli (Fig. 4a). The wild-type Arabidopsis T87 cells were also yellow when cultured in darkness; therefore, we did not observe a marked difference in color phenotype between the vector control and the transgenic lines (Fig. 4b). However, the aqueous extracts from transgenic lines were slightly yellow, whereas that from the vector control was colorless (Fig. 4c). HPLC analysis detected some very weak peaks in Arabidopsis transgenic line no. 1, but we could not identify specific betaxanthins.

These results indicated that the combined expressions of LeTYR and MjDOD could induce the accumulation of betaxanthins in cultured cells of non-Caryophyllales plants, but the specific components and amounts of betalains produced differed between tobacco and Arabidopsis.

\section{Discussion}

Betalains are Caryophyllales-specific plant pigments that are responsible for the red-violet and yellow coloration instead of anthocyanins in some plants in the order Caryophyllales. The betalain biosynthetic pathway in plants was deduced from genetic and metabolite analysis of $P$. grandiflora mutants ${ }^{23}$, and then the genes encoding DOD, betanidin 5GT, and $c$ DOPA5GT were identified from plants in the Caryophyllales ${ }^{8,11,22,24}$. More recently, CYP76AD1 encoding L-DOPA oxidase was identified from Beta vulgaris, and it was revealed that the oxidation of L-DOPA to cyclo-DOPA was catalyzed by this enzyme in betacyanin biosynthesis. It was suggested that this cytochrome P450, CYP76AD1, may have 
tyrosine-hydroxylating activity to synthesize L-DOPA from tyro$\sin ^{9}$, although further confirmation is necessary. Thus, the gene encoding an enzyme with tyrosine-hydroxylating activity, which is responsible for the crucial first step in betalain biosynthesis, was not identified, although the enzyme had been partially purified in earlier works ${ }^{13,14}$. The results of those previous studies strongly suggested that a tyrosinase belonging to the copper protein polyphenol oxidase (PPO) family is the tyrosine-hydroxylating enzyme involved in betalain biosynthesis, although it has never been proven conclusively. Most plant PPOs have a plastid transit peptide at the $\mathrm{N}$-terminal ${ }^{17}$. Although PPO genes have been cloned from pokeweed (Phytolacca americana), it is not certain whether they actually function in betalain biosynthesis ${ }^{25}$. Müeller et al. ${ }^{26}$ reported the purification of tyrosinase from the betalain-containing pileus of the mushroom Amanita muscaria, but no additional experiments had been conducted until now.

In previous studies, broad bean and $D O D$-expressing Arabidopsis produced betalains when fed with betalamic acid and L-DOPA, respectively ${ }^{10,19}$. These results suggested that anthocyanin-producing plant species have the potential to produce betalains if they are provided with suitable precursors for betalain biosynthesis. However, a betalain-specific gene that encodes an enzyme with tyrosinehydroxylating activity, whether from higher plants or fungi, was not available for combining with $D O D$ to complete the biosynthetic pathway. We considered that mushroom tyrosinases linked to the synthesis of melanin pigments have been well characterized ${ }^{17}$ and might be used to reconstruct betalain biosynthesis. In fact, LeTYR from shiitake mushroom resulted in accumulation of L-DOPA in BY2 cells; that is, the foreign LeTYR protein showed tyrosine-hydroxylating activity in cultured plant cells (Fig. 2c). Thereafter, MjDOD protein could convert L-DOPA produced by LeTYR into betalamic acid, leading to the formation of yellow betaxanthins in transgenic tobacco BY2 cells expressing both MjDOD and LeTYR genes (Fig. 2a; Fig. 3). The betaxanthin accumulation peaked at 8 weeks after subculture, even though the transgenic calli turned brown in prolonged culture after 5 weeks (Figs. 3a,b, Supplemental Fig. S1). This suggested that there was accumulation of other metabolites as a result of the foreign tyrosinase activity. Since the browning also occurred in transgenic BY2 cells expressing LeTYR only, these were not betalainderived compounds. This browning was considered to represent accumulation of L-DOPA-derived (but not betaxanthin-derived) metabolites via dopaquinone that might be toxic to plant cells. One strategy to reduce or eliminate browning is to convert the metabolites to nontoxic compounds by introducing other detoxifying enzymes. If successful, this may result in more effective accumulation of betaxanthins in transgenic BY2 cells. We also produced a transgenic Arabidopsis T87 cell line by introducing the same constructs (Fig. 4). However, betaxanthin accumulated to lower levels in T87 transgenic cells than in BY2 transgenic cells, and the coloration of Arabidopsis transgenic cells could not be observed visually (Fig. 4b). It is unknown why the amount of heterologous betaxanthin produced differed among the plant species used as transformation hosts, but there are several possible explanations: the transport efficiency of BA may differ between tobacco and Arabidopsis, and/or there may be differences in tyrosine availability or in competing endogenous reactions that use tyrosine and/or the L-DOPA substrate. Further research is required to elucidate the precise reason(s).

The condensation of BA with amino acids in planta is a spontaneous reaction ${ }^{10}$. Pro-Bx accumulated preferentially in transgenic BY2 cells, reaching a concentration of $3.3 \mu$ moles/g dry weight (Fig. $3 c$ ). Pro-Bx (indicaxanthin) accumulated as a major pigment in Opuntia ficus-indica ${ }^{27}$ and Mirabilis jalapa ${ }^{28}$. The yellow callus from B. vulgaris produced $4.2 \mu$ moles/g dry weight of total betaxanthin ${ }^{29}$. Therefore, the amounts of betaxanthins accumulated in transgenic BY2 cells were similar to those reported in calli and roots from plants in the Caryophyllales. When BA was fed to broad bean, the major betaxanthin was dopaxanthin ${ }^{10}$. In that case, amino acid analysis of the hypocotyl extract of broad bean seedlings revealed that L-DOPA was the most abundant amino acid present. However, the accumulation levels of amino acids are not the only factors influencing condensation reactions with $\mathrm{BA}^{30}$. Further studies are necessary to clarify the mechanisms of accumulation of specific betaxanthins in plant cells.

We transformed 35Sp-LeTYR/35Sp-MjDOD transgenic BY2 calli with a $c D O P A 5 G T$ gene from $M$. jalapa ${ }^{24}$. However, none of the transgenic cell lines accumulated betacyanins. This indicated that cyclo-DOPA, which is the substrate of cDOPA5GT, was not abundantly accumulated, probably because of deficient or low L-DOPA oxidase activity. It is noteworthy that C-terminal processing of tyrosinase has been reported in several fungi ${ }^{31-33}$. Processing of tyrosinase is catalyzed by endogenous proteases such as the serine proteases, and this proteolytic cleavage plays a critical role in enzyme activity. For example, analysis of a recombinant tyrosinase from Pholiota microspora revealed that the latent $67-\mathrm{kDa}$ premature form did not have catalytic activity but the C-terminal cleaved $44-\mathrm{kDa}$ mature form was catalytically active ${ }^{33}$, although the analysis did not distinguish between monophenolase and diphenolase activities. In transgenic BY2 calli expressing $L e T Y R$, the premature $68-\mathrm{kDa}$ LeTYR was detected from the early growth stages but the mature $45-\mathrm{kDa}$ LeTYR appeared at later growth stages (Fig. 2c, Supplementary Fig. S2). It is unknown why LeTYR was not efficiently processed, but it is likely that plant proteases could not efficiently process the fungal tyrosinase protein because of its low expression level at the early growth stages of BY2 cells. In light of these facts, it is most likely that the unprocessed LeTYR accumulated in BY2 cells had only tyrosine-hydroxylating activity, and not DOPA oxidase activity. However, betacyanins were not accumulated even at later growth stages when the mature $45-\mathrm{kDa}$ LeTYR appeared, suggesting that the enzymatic activity of the shiitake tyrosinase expressed in BY2 calli was insufficient for efficient production of cyclo-DOPA. Therefore, the additional expression of CYP76AD1 might induce betacyanin accumulation in transgenic BY2 calli. Moreover, Goldfeder et al. ${ }^{34}$ demonstrated that the amino acid residue at position 218 influenced the monophenolase/diphenolase activity ratio of a bacterial tyrosinase by affecting the positioning of substrates within the active site. Protein engineering of a fungal tyrosinase involved in enzymatic activity or C-terminal processing might be also useful for the effective production of betalains in plant cells.

In conclusion, our results demonstrated that it is possible to engineer betalain biosynthesis in a heterologous expression system without extraneous substrate feeding. A fungal tyrosinase was able to substitute for the tyrosine-hydroxylating enzyme of betaxanthinaccumulating plants to accumulate L-DOPA in plant cells. Betalains are naturally limited to the order Caryophyllales in higher plants, but genetic engineering can now overcome the species barrier. As betalain pigments have beneficial properties for human nutrition and plant coloration, our approach may contribute to the development of genetically modified crops in the future.

\section{Methods}

Vector construction and BY2 transformation. The coding region sequences (CDS) of $L$. edodes tyrosinase (LeTYR, accession number AB027512) $)^{21}$ and $M$. jalapa DOPA 4,5-dioxygenase (MjDOD, AB435372) 22 were used to replace the GUS gene in the binary vector $\mathrm{pBI} 121$ (kanamycin resistance) and pEBisHR (hygromycin resistance), respectively. pBI121-35Sp-LeTYR and pEBisHR-35Sp-MjDOD binary vectors were transformed into Agrobacterium tumefaciens EHA105 or EHA101.

The transformation of BY2 cells was performed following the procedure of $\mathrm{An}^{35}$ Transgenic calli were selected on medium containing $200 \mathrm{mg} \mathrm{l}^{-1}$ kanamycin or $20 \mathrm{mg} \mathrm{l}^{-1}$ hygromycin $\mathrm{B}$, and $500 \mathrm{mg} \mathrm{l}^{-1}$ carbenicillin, and were subcultured every 2 weeks. A. thaliana ecotype Columbia suspension-cultured $\mathrm{T} 87 \mathrm{cells}^{36}$ were obtained from the RIKEN BioResource Center. T87 cells were transformed by an Agrobacterium-mediated method as described by Ogawa et al. ${ }^{37}$.

Expression analysis. Total RNAs were isolated from $200 \mathrm{mg}$ (fresh weight) transgenic calli using a Fast RNA pro GREEN Kit (MP Bio Japan, Tokyo, Japan). 
Total RNAs $(5 \mu \mathrm{g})$ were separated on a $1 \%$ agarose gel, and then transferred onto a Hybond $\mathrm{N}^{+}$membrane (GE healthcare, Little Chalfont, UK). The probes for LeTYR and $M j D O D$ genes were labeled using a DIG-PCR labeling Kit (Roche Applied Sciences, Indianapolis, IN, USA). The hybridization and detection procedures were performed using the DIG Luminescent Detection Kit for Nucleic Acids (Roche) according to manufacturer's instructions.

Western blot analysis of LeTYR protein. Tissues ( $1 \mathrm{~g}$ fresh weight) of the transgenic BY2 and Arabidopsis T87 cell lines were lyophilized, and crude proteins were extracted using $400 \mu \mathrm{l} 50 \mathrm{mM}$ HEPES buffer [pH 7.0]. Protein concentration was measured as described by Bradford ${ }^{38}$. For each transgenic callus, $10 \mu \mathrm{g}$ crude protein was separated by SDS-PAGE (10\% acrylamide) and transferred onto a polyvinylidene difluoride (PVDF) membrane. The membrane was blocked by blocking buffer (phosphate buffered saline [PBS] plus 5\% skimmed milk and $0.3 \%$ Tween-20) for at least $12 \mathrm{~h}$ and then probed with a rabbit polyclonal antibody raised against LeTYR ${ }^{21}$. After extensive washing, membranes were incubated with the goat anti-rabbit IgG secondary antibody conjugated to alkaline phosphatase (Bio-Rad Laboratories, Hercules, CA, USA) and detected using an Immun-Blot Assay Kit (Bio-Rad).

Tyrosine-hydroxylating activity assay. To investigate tyrosine-hydroxylating activity, crude proteins were extracted from freeze-dried transgenic BY2 cells at 4 weeks after subculture using $400 \mu \mathrm{l} 50 \mathrm{mM}$ HEPES buffer [pH 7.0]. After centrifugation at $20,000 \times \mathrm{g}$ at $4^{\circ} \mathrm{C}$ for $10 \mathrm{~min}$, the supernatants were collected and protein concentrations were measured as described above. Then, $25 \mu$ l crude extract containing $150 \mu \mathrm{g}$ protein was mixed with $25 \mu \mathrm{l} 2 \mathrm{mM}$ tyrosine and incubated at $30^{\circ} \mathrm{C}$ for $30 \mathrm{~min}$. The reaction was terminated by incubating the mixture at $95^{\circ} \mathrm{C}$ for $3 \mathrm{~min}$. Boiled crude extracts were also assayed as the control. The mixture was centrifuged at $20,000 \times \mathrm{g}$ for $5 \mathrm{~min}$, and the supernatants were subjected to further analysis. The synthesized L-DOPA was quantified by capillary electrophoresis-mass spectrometry (CE-MS; Agilent Technologies, Santa Clara, CA, USA) according to the method of Takahashi et $\mathrm{al}^{39}$. The activities were represented by the amount of newly synthesized L-DOPA (nmol h${ }^{-1} \mathrm{mg}^{-1}$ protein). The MBTH assay was also performed to detect diphenolase activity as described by Rodriguez-Lopez et al. ${ }^{40}$.

Metabolite analysis. Transgenic BY2 cells (100 mg fresh weight) were powdered using a Micro Smash M100 (TOMY) and homogenized with $200 \mu \mathrm{l} 1 \%$ (v/v) formic acid. After centrifugation, the supernatant was filtered through a Millipore 5-kDacutoff filter (Millipore) and the filtrates were used for analysis. Betaxanthins were analyzed by Agilent 6520 Accurate-Mass quadrupole time-of-flight liquid chromatography/mass spectrometry (Q-TOF LC/MS; Agilent Technologies). Separation was performed on a Develosil PRAQUEOUS-AR-5 column $(4.6 \mathrm{~mm} \times$ $250 \mathrm{~mm}$; Numura Chemical Co., Seto, Japan) with a gradient elution of 3-20\% (v/v) acetonitrile containing $0.1 \%(\mathrm{v} / \mathrm{v})$ formic acid for $60 \mathrm{~min}$, followed by $20-60 \%(\mathrm{v} / \mathrm{v})$ for $30 \mathrm{~min}$ at a flow rate of $0.25 \mathrm{ml} \mathrm{min} 1^{-1}$ at $30^{\circ} \mathrm{C}$. Absorbance at $470 \mathrm{~nm}$ was monitored by an Agilent 1200 Diode-Array Detector SL (Agilent Technologies). The total concentration of betaxanthin was calculated using the molar extinction coefficient ( $480 \mathrm{~nm}, \varepsilon=48,000)^{29}$. Pro-Bx was quantified based on the area on the HPLC chromatogram monitored at $470 \mathrm{~nm}$ using synthetic authentic Pro-Bx as the standard. The HPLC separation was performed as described previously ${ }^{41}$.

1. Grotewold, E. The genetics and biochemistry of floral pigments. Annu. Rev. Plant Biol. 57, 761-780 (2006).

2. Tanaka, Y., Sasaki, N. \& Ohmiya, A. Biosynthesis of plant pigments: anthocyanins, betalains and carotenoids. Plant J. 54, 733-749 (2008).

3. Stafford, H. A. Anthocyanins and betalains: evolution of the mutually exclusive pathways. Plant Sci. 101, 91-98 (1994).

4. Hinz, U. G., Fivaz, J., Girod, P. A. \& Zyrd, J. P. The gene coding for the DOPA dioxygenase involved in betalain biosynthesis in Amanita muscaria and its regulation. Mol. Gen. Genet. 256, 1-6 (1997).

5. Clement, J. S. \& Mabry, T. J. Pigment evolution in the Caryophyllales: a systematic overview. Bot. Acta 109, 360-367 (1996).

6. Brockington, S. F., Walker, R. H., Glover, B. J., Soltis, P. S. \& Soltis, D. E. Complex pigment evolution in the Caryophyllales. New Phytol. 190, 854-864 (2011).

7. Strack, D., Vogt, T. \& Schliemann, W. Recent advances in betalain research. Phytochemistry 62, 247-269 (2003).

8. Christinet, L., Burdet, F. X., Zaiko, M., Hinz, U. \& Zryd, J. P. Characterization and functional identification of a novel plant 4,5-extradiol dioxygenase involved in betalain pigment biosynthesis in Portulaca grandiflora. Plant Physiol. 134, 265-274 (2004).

9. Hatlestad, G. J. et al. The beet $R$ locus encodes a new cytochrome $\mathrm{P} 450$ required for red betalain production. Nat. Genet. 44, 816-820 (2012).

10. Schliemann, W., Kobayashi, N. \& Strack, D. The decisive step in betaxanthin biosynthesis is a spontaneous reaction. Plant Physiol. 119, 1217-1232 (1999)

11. Vogt, T., Grimm, R. \& Strack, D. Cloning and expression of a cDNA encoding betanidin 5-O-glucosyltransferase, a betanidin- and flavonoid-specific enzyme with high homology to inducible glucosyltransferases from the Solanaceae. Plant J. 19, 509-519 (1999).

12. Sasaki, N., Adachi, T., Koda, T. \& Ozeki, Y. Detection of UDP-glucose:cycloDOPA 5-O-glucosyltransferase activity in four o'clocks (Mirabilis jalapa L.). FEBS Lett. 568, 159-162 (2004).
13. Steiner, U., Schliemann, W., Böhm, H. \& Strack, D. Tyrosinase involved in betalain biosynthesis of higher plants Planta 208, 114-124 (1999).

14. Steiner, U., Schliemann, W. \& Strack, D. Assay for tyrosine hydroxylation activity of tyrosinase from betalain-forming plants and cell cultures. Anal. Biochem. 238, 72-75 (1996).

15. Yamamoto, K., Kobayashi, N., Yoshitama, K., Teramoto, S. \& Komamine, A. Isolation and purification of tyrosine hydroxylase from callus cultures of Portulaca grandiflora. Plant Cell Physiol. 42, 969-975 (2001).

16. Luthra, P. M. \& Singh, S. Identification and optimization of tyrosine hydroxylase activity in Mucuna pruriens DC. var. utilis. Planta 231, 1361-1369 (2010).

17. van Gelder, C. W. G., Flurkeya, W. H. \& Wichers, H. J. Sequence and structural features of plant and fungal tyrosinases. Phytochemistry 45, 1309-1323 (1997).

18. Schliemann, W., Steiner, U. \& Strack, D. Betanidin formation from dihydroxyphenylalanine in a model assay system. Phytochemistry 49, 1593-1598 (1998).

19. Harris, N. N. et al. Betalain production is possible in anthocyanin-producing plant species given the presence of DOPA-dioxygenase and L-DOPA. BMC Plant Biol. 12, 34 (2012).

20. Nagata, T., Okada, K., Takebe, I. \& Matsui, C. Delivery of tobacco mosaic virus RNA into protoplasts mediated by reverse-phase evaporation vesicles (liposomes). Mol. Gen. Genet. 184, 161-165 (1981).

21. Sato, T. et al. The tyrosinase-encoding gene of Lentinula edodes, Letyr, is abundantly expressed in the gills of the fruit-body during post-harvest preservation. Biosci. Biotechnol. Biochem. 73, 1042-1047 (2009).

22. Sasaki, N. et al. Detection of DOPA 4,5-dioxygenase (DOD) activity using recombinant protein prepared from Escherichia coli cells harboring cDNA encoding DOD from Mirabilis jalapa. Plant Cell Physiol. 50, 1012-1016 (2009).

23. Trezzini, G. F. \& Zryd, J.-P. Portulaca grandiflora: a model system for the study of the biochemistry and genetics of betalain synthesis. Acta Horticul. 280, 581-585 (1990).

24. Sasaki, N. et al. Isolation and characterization of cDNAs encoding an enzyme with glucosyltransferase activity for cyclo-DOPA from four o'clocks and feather cockscombs. Plant Cell Physiol. 46, 666-670 (2005).

25. Joy, R. W., Sugiyama, M., Fukuda, H. \& Komamine, A. Cloning and characterization of polyphenol oxidase cDNAs of Phytolacca americana. Plant Physiol. 107, 1083-1089 (1995).

26. Müeller, L. A., Hinz, U. \& Zrÿd, J.-P. Characterization of a tyrosinase from Amanitamuscaria involved in betalain biosynthesis. Phytochemistry 42, 15111515 (1996).

27. Stintzing, F. C., Schieber, A. \& Carle, R. Identification of betalains from yellow beet (Beta vulgaris L.) and cactus pear [Opuntia ficus-indica (L.) Mill.] by highperformance liquid chromatography-electrospray ionization mass spectrometry. J. Agric. Food Chem. 50, 2302-2307 (2002).

28. Piattelli, M., Minale, L. \& Nicolaus, R. A. Pigments of centrospermae-V. Betaxanthins from Mirabilis jalapa L. Phytochemistry 4, 817-823 (1965).

29. Girod, P. A. \& Zryd, J. P. Secondary metabolism in cultured red beet (Beta vulgris L.) cells: Differential regulation of betaxanthin and betacyanin biosynthesis. Plant Cell Tiss. Org. Cult. 25, 1-12 (1991).

30. Böhm, H. \& Mäck, G. Betaxanthin formation and free amino acids in hairy roots of Beta vulgaris var. lutea depending on nutrient medium and glutamate or glutamine feeding. Phytochemistry 65, 1361-1368 (2004).

31. Wichers, H. J. et al. Cloning, expression and characterisation of two tyrosinase cDNAs from Agaricus bisporus. Appl. Microbiol. Biotechnol. 61, 336-341 (2003)

32. Selinheimo, E. et al. Production and characterization of a secreted, C-terminally processed tyrosinase from the filamentous fungus Trichoderma reesei. FEBS $J$. 273, 4322-4335 (2006).

33. Kawamura-Konishi, Y., Maekawa, S., Tsuji, M. \& Goto, H. C-terminal processing of tyrosinase is responsible for activation of Pholiota microspora proenzyme. Appl. Microbiol. Biotechnol. 90, 227-234 (2011).

34. Goldfeder, M., Kanteev, M., Adir, N. \& Fishman, A. Influencing the monophenolase/diphenolase activity ratio in tyrosinase. Biochim. Biophys. Acta 1834, 629-633 (2013)

35. An, G. High efficiency transformation of cultured tobacco cells. Plant Physiol. 79, 568-570 (1985)

36. Axelos, M., Curie, C., Mazzolini, L., Bardet, C. \& Lescure, B. A protocol for transient gene expression in Arabidopsis thaliana protoplasts isolated from cell suspension cultures. Plant Physiol. Biochem. 30, 123-128 (1992).

37. Ogawa, Y. et al. Efficient and high-throughput vector construction and Agrobacterium-mediated transformation of Arabidopsis thaliana suspensioncultured cells for functional genomics. Plant Cell Physiol. 49, 242-250 (2008)

38. Bradford, M. M. A rapid and sensitive method for the quantitation of microgram quantities of protein utilizing the principle of protein-dye binding. Anal. Biochem. 72, 248-254 (1976)

39. Takahashi, H., Uchimiya, H. \& Hihara, Y. Difference in metabolite levels between photoautotrophic and photomixotrophic cultures of Synechocystis sp. PCC 6803 examined by capillary electrophoresis electrospray ionization mass spectrometry. J. Exp. Bot. 59, 3009-3018 (2008).

40. Rodriguez-Lopez, J. N., Escribano, J. \& Garcia-Canovas, F. A continuous spectrophotometric method for the determination of monophenolase activity of tyrosinase using 3-methyl-2-benzothiazolinone hydrazone. Anal. Biochem. 216, 205-212 (1994) 
41. Sekiguchi, H., Ozeki, Y. \& Sasaki, N. In vitro synthesis of betaxanthins using recombinant DOPA 4,5-dioxygenase and evaluation of their radical-scavenging activities. J. Agric. Food Chem. 58, 12504-12509 (2010).

\section{Acknowledgments}

We thank Dr. Toshitsugu Sato, Kitami Institute of Technology, for providing the shiitake tyrosinase gene and for helpful discussions. The authors thank Dr. Ikuo Nakamura, Chiba University, for providing the pEBis binary vector. We thank the RIKEN BioResource Center for providing Arabidopsis thaliana T87 cells.

\section{Author contributions}

T.N. and M.N. designed research, T.N., E.Y., H.T., T.I., N.S., M.S., I.T., M.S., Y.S. and M.N. performed research, T.N., H.T., N.S., Y.O. and M.N. analyzed data, and T.N. and M.N. wrote the paper.

\section{Additional information}

Supplementary information accompanies this paper at http://www.nature.com/ scientificreports

Competing financial interests:The authors declare no competing financial interests.

How to cite this article: Nakatsuka, T. et al. Genetic engineering of yellow betalain pigments beyond the species barrier. Sci. Rep. 3, 1970; DOI:10.1038/srep01970 (2013).

(c) (i) $(-)$ This work is licensed under a Creative Commons Attribution-

BY NC ND NonCommercial-NoDerivs Works 3.0 Unported license. To view a copy of this license, visit http://creativecommons.org/licenses/by-nc-nd/3.0 